\title{
Diagnostic accuracy of pre-treatment biopsy for grading cutaneous mast cell tumors in
} dogs

T. Shaw ${ }^{1 *}$, S. T. Kudnig ${ }^{1}$, S. M. Firestone ${ }^{2}$

${ }^{1}$ Melbourne Veterinary Specialist Centre, Glen Waverley, VIC, Australia

${ }^{2}$ Asia-Pacific Centre for Animal Health, Faculty of Veterinary and Agricultural Sciences, The University of Melbourne, Parkville, VIC, Australia

*Corresponding author: 70 Blackburn Road, Glen Waverley, VIC 3150, Australia; shaw.tania@gmail.com

\begin{abstract}
Mast cell tumors (MCTs) are common tumors of the canine skin, and are estimated to represent up to $20 \%$ of all skin tumors in dogs. Tumor grade has a major impact on the incidence of local recurrence and metastatic potential. In addition to helping the clinician with surgical planning, knowledge of the tumor grade also assists in proper prognostication and client education. For pre-treatment biopsies to be useful, there must exist a high level of correlation between the histopathological grade obtained from the pre-treatment biopsy and the actual histopathological grade from the excisional biopsy. The aim of this study was to determine concordance of tumor grade between various biopsy techniques (wedge, punch, needle core) and the 'gold standard' excisional biopsy method. We found an overall concordance rate of $96 \%$ based on the Patnaik grading system, and an overall concordance rate of $92 \%$ based on the Kiupel grading system. The accuracy of the various biopsy techniques (wedge, punch and needle core) when compared to excisional biopsy, was 92, 100
\end{abstract}

This is the author manuscript accepted for publication and has undergone full peer review but has not been through the copyediting, typesetting, pagination and proofreading process, which may lead to differences between this version and the Version of Record. Please cite this article as doi: $10.1111 /$ vco.12346

This article is protected by copyright. All rights reserved. 
and $100 \%$ respectively based on the Patnaik grading system, and 90, 95 and $100 \%$ respectively based on the Kiupel grading system. Of the cases with discordant results, the pre-treatment biopsies tended to underestimate the grade of the tumor. Based on these results, we conclude that pre-treatment biopsies are sufficiently accurate for differentiating low grade from high grade mast cell tumors, regardless of biopsy technique or tumor location.

Keywords: biopsy, mast cell tumor, grade

\section{Introduction}

Mast cell tumors (MCTs) are common tumors of the canine skin, and are estimated to represent up to $20 \%$ of all skin tumors in dogs. ${ }^{1-15}$ Boxers, Bulldogs and Pugs are at higher risk of developing MCTs, however these breeds tend to have less aggressive (low- or intermediate-grade) tumors. ${ }^{1,3}$ Shar-Peis and Labrador Retrievers tend to have more aggressive tumors, and Golden Retrievers are at risk of developing multiple tumors. ${ }^{1,3,7}$

Mast cell tumors most commonly occur in the dermis and subcutaneous tissue, however they are occasionally reported in extra-cutaneous sites, such as the conjunctiva, salivary gland, nasopharynx, larynx, oral cavity, gastrointestinal tract, urethra and spine. ${ }^{3}$ Visceral MCTs (disseminated or systemic mastocytosis) are almost always preceded by an undifferentiated primary cutaneous tumor, and primary mast cell leukemia is extremely rare. ${ }^{3}$ Cutaneous MCTs are most common on the trunk (48-65\%), followed by the extremities (25-47\%), and 
the head and neck (10-13\%). ${ }^{3,8-10,12}$ In general, MCTs are more likely to affect the posterior part of the body (hindlimbs, perineum and prepuce). ${ }^{3,9,10,12}$

Solitary cutaneous masses are most common, however $10-30 \%$ of dogs present with multiple masses that may occur synchronously or sequentially., ${ }^{3,9,10,12,16}$ Low grade, well-differentiated MCTs usually present as a solitary, slow-growing cutaneous nodule, which may have been present for more than 6 months prior to presentation. ${ }^{1,3,12}$ High grade, undifferentiated MCTs tend to grow rapidly, ulcerate, cause considerable irritation, and may give rise to smaller satellite nodules in the surrounding tissues. ${ }^{3,12}$

Clinical signs associated with MCTs are caused by the local release of histamine, heparin and other vasoactive amines from tumor cells, and granule release from invading eosinophils. ${ }^{1,3,12}$ This may cause the mass to fluctuate in size and degree of redness, due to local vasodilation and intratumoral edema (termed Darier's sign). ${ }^{1,3,7,12}$ Gastrointestinal ulceration is another potential complication of MCTs, therefore signs such as vomiting, hematochezia, anorexia, melena and abdominal pain may be observed occasionally. ${ }^{1,3,12}$ The pathogenesis of gastrointestinal ulceration associated with MCTs appears to be caused by a combination of vascular damage, excessive gastric acid production through stimulation of $\mathrm{H}_{2}$ receptors on parietal cells, and hypermotility, and such complications are usually restricted to the aggressive high-grade or extensive MCTs. ${ }^{3}$ 
The etiology of MCTs in dogs is unknown. Recent studies have implicated the stem cell factor receptor, KIT, in the etiology of canine MCTs, as KIT expression has been demonstrated immunohistochemically in both normal and neoplastic canine mast cells, with higher expression in poorly differentiated MCTs. ${ }^{3}$ Aberrant cytoplasmic KIT expression due to $c$-kit mutations has been associated with poorer clinical outcome, and previous studies have shown that $15-40 \%$ of all canine MCTs are affected by $c$-kit mutations. ${ }^{3}$

Tumor grade has a major impact on the expected biologic behavior of MCTs, including the incidence of local recurrence and metastatic potential. Well-differentiated low grade tumors have a metastatic rate of $<10 \%$, whereas intermediate grade tumors are considered low to moderate in metastatic potential, and undifferentiated high grade tumors have a much higher metastatic rate, ranging from $55-96 \% .^{3,5-7,9,13}$ Of the MCTs that do metastasize, most of them first metastasize to the local lymph nodes, then to the spleen (46\%), liver (41\%), and other visceral organs. ${ }^{3,9,15}$ Historically, histopathological grade has been one of the most reliable predictors of survival in dogs with cutaneous MCTs. ${ }^{3,5-7,9-14}$ Mitotic index has also been shown to correlate with MCT grade, development of metastatic disease, and survival time. ${ }^{6}$

For many years, the system most commonly used to grade MCTs was a 3-tiered scheme described by Patnaik and colleagues. The Patnaik system classifies MCTs as grade I (low), II (intermediate), or III (high) depending upon several histopathological features that include cellularity, cellular morphology, invasiveness, mitotic activity, and stromal reaction. ${ }^{11}$ With this grading system, grade I tumors are generally associated with an excellent prognosis and 
are usually cured by complete surgical excision exclusively. ${ }^{4,5,7,11,13}$ Grade III tumors are predicted to be more biologically aggressive, with greater risk of invasion and distant metastasis, and often require adjunctive chemotherapy along with surgical excision. ${ }^{4,5,7,11,13}$ Unfortunately, grade II MCTs are overrepresented compared to grade I and grade III MCTs, and their behavior is difficult to predict - some of these tumors behave in a benign fashion, like grade I tumors, while some behave aggressively, like grade III tumors, and 5-22\% of these tumors will metastasize. ${ }^{4,5,7,11,13,15}$ There is a tendency for pathologists to opt for a grade II when presented with a tumor that is borderline between a grade I and a grade II, making the ambiguity of a grade II MCT of little prognostic value. ${ }^{14}$ The Patnaik system has been shown to have wide interobserver variability, with 50-60\% discordance among experienced pathologists when using the Patnaik grading criteria. ${ }^{4,5,8,11,13-15,17}$ Sabattini and colleagues found that dogs with grade III MCTs had significantly shortened survival times than those with grade I or grade II tumors, however there were no statistically significant differences between grade I and grade II MCTs. ${ }^{11}$ They also found that only $12 \%$ of the dogs with grade II MCTs died from the disease.

To address the limitations posed by the Patnaik system, Kiupel and colleagues recently proposed a new 2-tiered grading scheme, which classifies MCTs as either low or high grade based on simple microscopic criteria, with more definitive categorization criteria including number of mitotic figures in combination with karyomegaly, multinucleated cells, or bizarre nuclei. ${ }^{8,11,14,17}$ This novel grading system demonstrated a 96-98\% consistency among pathologists and a high prognostic power. ${ }^{11,17}$ The prognosis for dogs following surgical 
removal of low grade and high grade mast cell tumors is starkly disparate. Sabattini and colleagues found that by the combined used of both grading systems, the Kiupel system allowed proper differentiation of the Patnaik grade II MCTs with good long-term prognosis from the small subset (14\%) of grade II MCTs that behaved more aggressively. ${ }^{11}$ They also found that the biologic behavior of Kiupel low grade MCTs was almost invariably benign, regardless of whether they were graded as grade I or grade II based on the Patnaik system, however among the Kiupel high grade MCTs, there was a distinct difference between the Patnaik grade II and grade III tumors, with significantly longer survival times observed with the grade II tumors.

Surgery is generally the mainstay of treatment for MCTs, and appropriate staging diagnostics should be considered before determining the perioperative course of therapy. ${ }^{9,10,12}$ The goal of surgery is complete tumor excision with tumor-free margins. ${ }^{10,18}$ All grade I and most grade II MCTs will be completely excised if resected with $2 \mathrm{~cm}$ lateral margins and one fascial plane deep. ${ }^{17}$ The recommendation for high grade MCTs is to obtain $3 \mathrm{~cm}$ lateral margins and one fascial plane deep, however the precise origin of the $3 \mathrm{~cm}$ margin recommendation is unclear. ${ }^{8,17}$ Recent studies have suggested that less aggressive surgery may suffice for many MCTs, specifically low grade MCTs. ${ }^{8-10}$ The ability to distinguish between a low and a high grade MCT preoperatively would significantly contribute to the prediction of prognosis and the decision to perform a radical resection or not. ${ }^{5}$ 
In addition to helping the clinician with surgical planning, knowledge of the tumor grade assists in proper prognostication and client education. ${ }^{19}$ As previously discussed, high grade MCTs are more likely to metastasize. Dogs with grade I MCTs are likely to experience longterm survival, whereas dogs with grade III MCTs have a reported median survival time of 6 months. ${ }^{13}$ In a study by Patnaik and colleagues, the survival rate of dogs with grade II tumors was 5 times that of dogs with grade III tumors. ${ }^{2}$

With such a large emphasis on the results of pre-treatment biopsy for surgical planning and prognostication, a high correlation between the histopathological grade obtained from the pretreatment biopsy and the actual histopathological grade is essential. An incorrectly graded 'low grade' MCT on a distal extremity on a pre-treatment biopsy sample, for example, may be treated inadequately with marginal excision followed by close monitoring for recurrence due to difficulties in wound closure in that location, putting the patient at a higher risk of recurrence and metastasis.

Historically, wedge biopsies have been considered to be the gold standard biopsy method for soft tissue mass diagnosis, due to the ability to obtain a relatively large sample while directly visualizing the tissue being obtained. ${ }^{19}$ However, minimally invasive and less expensive methods of biopsy such as needle core (Tru-Cut) biopsies are often performed with a similar intent of obtaining the diagnosis and grade of a tumor. ${ }^{19}$ Needle core biopsy samples have the advantage of being able to be performed under sedation and local anesthesia with minimal morbidity to the patient. ${ }^{19}$ Disadvantages of incisional biopsies compared to fine-needle 
aspirate cytology are the risk of delayed wound healing due to the release of proteolytic enzymes, vasoactive amines and histamine, local hemorrhage caused by the release of heparin from the MCT granules, and increased cost. ${ }^{1,3,7,12}$ Killick and colleagues evaluated the effect of mast cell tumor excision on wound healing, and found that 5 out of 12 MCTs that were biopsied had delayed wound healing. ${ }^{20}$ However, delayed wound healing and wound dehiscence are of questionable significance, given that most cases would undergo tumor excision within several days of the biopsy. Additionally, pre-treatment with a $\mathrm{H}_{1}$ blocker may be considered prior to biopsy to prevent the effects of histamine on fibroblast activity during wound healing.

Traditionally, histologic grade could not be assessed by cytologic evaluation of fine-needle aspirates. $^{3,12}$ The grade of a MCT is determined by the characteristics of the neoplastic cells, such as degree of granulation, and cytologic and nuclear pleomorphism, number of mitotic figures, and extent of tumor invasion into the underlying tissues, which cannot be determined from cytologic evaluation of fine-needle aspirates. ${ }^{12}$ However, more recent studies have evaluated the use of fine-needle aspirate cytology for grading MCTs, with one study developing a unique cytologic grading system based on the Kiupel grading scheme, which is based solely on cellular features that could hypothetically evaluated on a cytological specimen. ${ }^{5,6}$ These studies have suggested that fine-needle aspirate cytology can provide a useful suggestion of the likely grade of the tumor. 
Camus and colleagues found that cytology was useful for predicting the behavior of canine cutaneous MCTs when adequately cellular samples were evaluated, based on a two-tier grading scheme developed by the authors. ${ }^{5}$ This study, however, found a higher false positive rate on cytology than histology, which could result in more aggressive surgery than necessary. They concluded that their proposed cytologic grading system correlated well with the histologic grading system, with cytologically low grade tumors having prolonged survival and low likelihood of being histologically high grade. Scarpa and colleagues investigated the possibility of applying the Kiupel grading system to accurately grade cutaneous MCTs on cytological smears, and found that the histological grade was correctly predicted in $94 \%$ of cases. ${ }^{6}$ This study, however, found that the Kiupel system tended to underestimate the grade of MCTs when applied to cytological smears, as it failed to distinguish high grade MCTs in $4 \%$ of cases, giving it a $85 \%$ sensitivity. Scarpa and colleagues found that the highest disparities between histology and cytology were due to the low number of mitoses detected in the cytological specimens, which is a frequent occurrence in cytology. ${ }^{6}$ They concluded that while their findings supported a role for cytological grading in canine MCTs, it did not support the application of the Kiupel grading system to cytopathology as a precise and effective method, although in the majority of cases it can provide helpful insights to assist in clinical decisions.

To our knowledge, there has been no study evaluating the diagnostic quality of pre-treatment incisional (wedge, punch or needle core) biopsies for grade determination of MCTs and their correlation with the actual histopathological grade from excisional biopsies for surgical 
treatment of MCTs. The diagnostic quality of needle core versus wedge biopsy for grade determination in cutaneous neoplasms in humans has been assessed. Heslin and colleagues found that needle core biopsy accurately determined high versus low grade in $93 \%$ of cases, compared to a $97 \%$ accuracy noted with wedge biopsies. ${ }^{21}$ To our knowledge, no similar study in animals has validated the concordance of any biopsy technique with regard to grade of MCT, nor compared the accuracy of various techniques in this regard.

The first aim of our study was to determine concordance of tumor grade between various biopsy techniques and the 'gold standard' excisional biopsy method. The secondary aim was to determine whether tumor location affected the accuracy of grading.

We hypothesized that there would be a strong concordance, in general, between the grade of pre-treatment and excisional biopsies, and that concordance between pre-treatment and excisional biopsies would be similar regardless of tumor location.

\section{Materials and methods}

Histopathology reports from the Australian Specialised Animal Pathology (ASAP) Laboratory were searched from 2008 to 2016 . Cases were included if the following criteria were met: (1) complete histopathology report for both pre-treatment biopsy and excisional biopsy, including tumor location; and (2) a diagnosis of MCT and the assignment of a 
histologic grade for both biopsy samples. Where cases had more than one sample recorded, only the first sample was included.

We estimated that we would require a minimum sample size of 93 dogs to have $95 \%$ confidence of estimating concordance to an absolute precision of $\pm 10 \%$, with the epiR library ${ }^{22}$, assuming a concordance of $59 \%$ (in the absence of prior published data, based on the findings of a similarly structured study of soft tissue sarcomas in dogs). ${ }^{19}$

Data from each histopathology report were entered into a commercially available database (Excel, Microsoft Corp, WA, USA). Data recorded, when available, included breed (categorized for analysis into the Australian National Kennel Council Ltd categories), age (in 6-month age groups), pre-treatment biopsy technique (wedge, punch, or needle core), histologic grade (Patnaik and/or Kiupel), tumor location (categorized into head and neck, trunk, extremities or unknown), tumor size, and biopsy date. For cases reported as a grade range, the higher of the grades was selected.

The level of agreement between the pre-treatment biopsy and the excisional biopsy was evaluated using the Kappa statistic, which measures the level of concordance between various assessments adjusted for concordance to occur by chance. ${ }^{23}$ The $\kappa$ value ranges from -1 to 1 , where higher values indicate more concordant ratings. Values $>0.75$ were considered excellent, values from 0.4 to 0.75 were considered fair to good, and values $<0.4$ were considered poor. ${ }^{19}$ Where appropriate, prevalence-adjusted-bias-adjusted $\kappa($ PABAK) was 
calculated. ${ }^{24}$ All $P$ values were two-sided, and $P<0.05$ was considered to indicate statistical significance. Where appropriate, Fisher's exact test was calculated for frequency tables, and confidence intervals for proportions were estimated using the Wilson score method in epiR. Univariable and multivariable logistic regression analyses were undertaken to compare concordance between subgroups based on clinical characteristics (pre-treatment biopsy technique, tumor location and tumor size) and signalment (age and breed categories). Statistical analyses were performed in the R statistical package ${ }^{25}$ and Stata version 14.1 (StataCorp, College Station, TX, USA).

\section{Results}

A total of 95 cases met the inclusion criteria, however 5 samples were from previously identified dogs and these were excluded, leaving a study sample size of 90 dogs. The median age of dogs in this study was 8 years (range $1-15$ years). The most common breeds represented in this study included: Labrador Retriever $(n=23)$, Staffordshire Bull Terrier $(n=$ 10), Boxer $(n=6)$, Pug $(n=5)$ and Jack Russell Terrier $(n=4)$.

A wedge biopsy was performed in 48 cases, a punch biopsy was performed in 38 cases, and a needle core biopsy was performed in 4 cases. All 90 cases were graded by both the Patnaik and the Kiupel systems. 
Based on the Patnaik system, the histologic grade of the pre-treatment biopsy samples was grade I in 5 cases $(6 \%)$, grade II in 82 cases $(91 \%)$, and grade III in 3 cases $(3 \%)$. The histologic grade of the excisional biopsy samples was grade I in 1 case (1\%), grade II in 86 cases $(96 \%)$, and grade III in 3 cases $(3 \%)$.

Based on the Kiupel system, the histologic grade of the pre-treatment biopsy samples was low grade in 78 cases $(87 \%)$ and high grade in 12 cases $(13 \%)$. The histologic grade of the excisional biopsy samples was low grade in 71 cases (79\%) and high grade in 19 cases $(21 \%)$.

Based on the Patnaik grades, the overall concordance between pre-treatment and excisional biopsy samples was $96 \%$ (86/90 samples in agreement). The level of agreement based on this grading system was assessed as good $(\kappa=0.65 ; 95 \%$ CI: $0.34,0.97)$, driven down by the high expected proportion of agreement (with $\mathrm{PABAK}=0.91$; representing excellent agreement). The concordance for the various pre-treatment biopsy techniques was $92 \%$ (44/48, 95\% CI: $80,97 \%)$ for wedge biopsies, $100 \%$ (38/38, 95\% CI: 91, 100\%) for punch biopsies, and 100\% (4/4, 95\% CI: 51, 100\%) for needle core biopsies, with little difference when considered by biopsy technique $(P=0.27)$. The four discordant results were all due to pre-treatment biopsies underestimating the grade (biopsies recorded as grade I on the pre-treatment biopsy sample but determined to be grade II on final histopathology of the excisional biopsy sample).

Based on the Kiupel grades, the overall concordance between pre-treatment and excisional biopsy samples was $92 \%$ (83/90 samples in agreement). The level of agreement based on this 
grading system was assessed as good $(\kappa=0.73 ; 95 \%$ CI: $0.54,0.92)$, and excellent considering $\mathrm{PABAK}=0.84$. The concordance rate for the various pre-treatment biopsy techniques was $90 \%$ (43/48, 95\% CI: 78, 95\%) for wedge biopsies, $95 \%$ (36/38, 95\% CI: 83 , $99 \%$ ) for punch biopsies, and $100 \%$ (4/4, 95\% CI: 51, 100\%) for needle core biopsies, with little difference when considered by biopsy technique $(P=0.61)$. Again, all seven of the inaccurate pre-treatment biopsies underestimated the grade, and all seven of these pretreatment biopsies were recorded as low grade on the pre-treatment biopsy sample but were determined to be high grade on final histopathology of the excisional biopsy sample. When cross-tabulated (Table 1), overrepresentation of Patnaik grade II tumors is apparent, with some tumors from this grade falling into the Kiupel low grade category and others the Kiupel high grade category.

Tumor location data were available for 80 of 90 cases $(89 \%)$. Ten (11\%) of the tumors were located on the head or neck, $35(38 \%)$ were located on the trunk, and $35(38 \%)$ were located on the extremities. Based on the Patnaik grades, the concordance rates between pre-treatment and excisional biopsies for tumors of the head and neck, trunk, and extremities was highly comparable regardless of tumor location ( $9 / 10$ tumors on the head and neck, 34/35 tumors on the trunk, and 33/35 tumors on the extremities were accurately graded based on their pretreatment biopsy samples; $P=0.49$ ). Similarly, high concordance was observed, irrespective of tumor location, based on the Kiupel grades (9/10 tumors on the head and neck, 32/35 tumors on the trunk, and 32/35 tumors on the extremities were accurately graded based on their pre-treatment biopsy samples; $P=1.00$ ). 
There was evidence that with increasing age of cases concordance decreased: for each year of increasing age, concordance based on the Patnaik system decreased by $39 \%$, and concordance based on the Kiupel system decreased by $21 \%$ (see Table 2). For the Patnaik grades, there was less concordance for smaller tumors (a doubling in tumor size appeared associated with a 7-fold increase in concordance). Concordance did not depend on tumor size based on the Kiupel system, or on breed or tumor location irrespective of grading system. Of all the breeds, the breeds categorized by the Australian National Kennel Council Ltd as terriers appeared to have a lower concordance rate when graded by the Patnaik system ( $86 \%)$, and a higher concordance rate when graded by the Kiupel system (91\%).

The duration of time between pre-treatment biopsy and excisional biopsy ranged from 2 days to 111 days, with a mean duration of 14 days, and a median duration of 9 days. Eight cases had the excisional biopsy performed less than 5 days after the pre-treatment biopsy, and 6 cases had the excisional biopsy performed more than 30 days after the pre-treatment biopsy.

\section{Discussion}

With such a large emphasis on the results of pre-treatment biopsy for surgical planning and prognostication, a high correlation between the histopathological grade obtained from the pretreatment biopsy and the actual histopathological grade is essential. In this study, we found a very high level of concordance in determining grade when the Patnaik grading system was 
used, and a high concordance rate when the Kiupel grading system was used. Importantly, although the Patnaik system has three grades, it is not considered to differentiate well between benign and aggressive grade II MCTs, and their behavior is difficult to predict. Grade II MCTs are known to be overrepresented, as is evident in this study, with $91 \%$ of the cases graded as grade II MCTs.

This study found that discordance in grading was more likely to underestimate rather than overestimate the grade of the tumor. This is important considering that a MCT in a location with limited tissue available for closure of a large defect, that has been incorrectly graded 'low grade' on a pre-treatment biopsy sample, may be treated inadequately with marginal excision followed by close monitoring for recurrence, putting the patient at a higher risk of recurrence and metastasis. This finding is similar to that found by Scarpa and colleagues, who investigated the possibility of applying the Kiupel grading system to accurately grade cutaneous MCTs on cytological smears. ${ }^{6}$ One possible explanation for this underestimation when using the Kiupel system is that the presence of mast cell granules may obscure nuclear details in well-differentiated MCTs, or the nuclei may be poorly stained, making it difficult to define their morphology. ${ }^{6}$ Therefore, based on the results presented here, where discordance was more likely to underestimate rather than overestimate the grade of the tumor, it would be advisable to err on the side of caution and use wider margins in locations amenable to reconstruction, even if the pre-treatment biopsy has been graded 'low grade'. 
This study also evaluated the diagnostic concordance of various pre-treatment biopsy techniques with regard to grade. All four of the discordant biopsies in the Patnaik system involved pre-treatment wedge biopsies, and all four of these underestimated the grade. Similarly, five out of the seven discordant biopsies in the Kiupel system involved wedge biopsies, with the other two involving punch biopsies, and all seven of these underestimated the grade as well. This finding was unexpected as historically, wedge biopsies have been considered to be the gold standard biopsy method for soft tissue mass diagnosis, due to the ability to obtain a relatively large sample while directly visualizing the tissue being obtained. However, this result was not statistically significant and could be explained by the low number of tumors evaluated using the needle core biopsy technique. Additionally, the retrospective nature of this study makes it difficult to determine where within the tumor mass the samples were taken from and how many samples were taken from each tumor. Greater intra-tumoral heterogeneity within large tumors would be expected (e.g. necrotic centers).

The location of the tumor did not have a statistically significant effect on the accuracy of the pre-treatment biopsy grade compared to the final histopathology grade of the excisional biopsy sample, irrespective of the grading system used. We observed that MCTs were more common on the trunk (39\%) and extremities (39\%) than on the head and neck $(11 \%)$, which is similar to the findings from previous reports.

This study has several limitations. One that may have led to an overestimation of the accuracy of the various pre-treatment biopsy techniques is related to the inclusion criteria for 
this study (i.e. cases must be diagnosed as MCT on both the pre-treatment biopsy and the excisional biopsy). It would be interesting to perform further research to evaluate the accuracy of both grading systems including cases that are diagnosed as MCTs on either the pre-treatment biopsy or the final excisional biopsy sample, but not the other.

Secondly, as previously mentioned, the retrospective nature of this study makes it difficult to determine where within the tumor mass the biopsies were taken from, and how many samples were taken from each tumor, which could affect the accuracy of the biopsy techniques.

Thirdly, due to the retrospective nature of this study, we did not have data available on whether the patients had received any treatments with corticosteroids or chemotherapy between the time of the pre-treatment biopsy and the excisional biopsy, and there was variability in the duration of time between the pre-treatment biopsy and the excisional biopsy. The administration of corticosteroids or chemotherapy between the time of pre-treatment biopsy and excisional biopsy could have the potential of altering the histological appearance of the tumor, however if this were true, then we would expect the pre-treatment biopsy to overestimate the grade, rather than underestimate it. Similarly, if the excisional biopsy was performed soon after the pre-treatment biopsy, it is possible that local inflammation and other complications associated with the pre-treatment biopsy itself could potentially lead to alterations to the histological appearance of the tumor, resulting in the excisional biopsy having a higher histological grade. However, only 8 out of 90 cases had the excisional biopsy performed less than 5 days after the pre-treatment biopsy in this study. Likewise, if the 
duration between the pre-treatment biopsy and the excisional biopsy was prolonged, there is the possibility that the tumor may have progressed from a low grade MCT to a high grade MCT by the time of the excisional biopsy. However, only 6 out of 90 cases had the excisional biopsy performed more than 30 days after the pre-treatment biopsy in this study.

Finally, the pre-treatment biopsy samples and the excisional biopsy samples in this study were reviewed by multiple unblinded pathologists, lending it to the potential for interobserver variability in grading, particularly with the Patnaik grading system, as previously addressed. However, in the clinical setting, pre-treatment biopsy samples and excisional biopsy samples are often read by different pathologists. We elected not to have the samples reviewed by a single pathologist because it does not represent a true clinical scenario where multiple pathologists are often involved in any chosen case.

In summary, this study evaluated the concordance of tumor grade between various biopsy techniques, considering the excisional biopsy method as a 'gold standard'. Pre-treatment biopsies were found to be an accurate method of differentiating low grade from high grade mast cell tumors, regardless of biopsy technique or tumor location. Unfortunately, without data on the actual size of the pre-treatment biopsy samples, and the small number of needle core biopsy samples available in this study, we cannot evaluate the effect of biopsy sample size on the accuracy of histological grading, and we cannot conclude that needle core biopsy is equivalent to wedge and punch biopsy techniques. The grade obtained from the pretreatment biopsy is important for surgical planning and prognostication purposes. However, 
the grade obtained from a pre-treatment biopsy in general should always be interpreted with caution, as there is still a risk that it may underestimate the grade of the tumor, leading to inadequate treatment in some cases.

\section{Acknowledgements}

We wish to thank Carl Muhlnickel, Libby Browne and Brad Galgut at ASAP Laboratory for assisting with searching of the database for relevant records. 


\section{References}

1. Dobson JM and Scase TJ. Advances in the diagnosis and management of cutaneous mast cell tumours in dogs. Journal of Small Animal Practice 2007; 48: 424-431. doi: 10.111/j.1748-5827.2007.00366.x.

2. Patnaik AK, Ehler WJ and MacEwen EG. Canine cutaneous mast cell tumor: morphologic grading and survival time in 83 dogs. Veterinary Pathology 1984; 21: 469474. doi: $10.1177 / 030098588402100503$.

3. Welle MM, Bley CR, Howard J and Rüfenacht S. Canine mast cell tumours: a review of the pathogenesis, clinical features, pathology and treatment. Veterinary Dermatology 2008; 19: 321-339. doi: 10.1111/j.1365-3164.2008.00694.x.

4. Camus MS, Priest HL, Koehler JW, et al. Cytologic criteria for mast cell tumor grading in dogs with evaluation of clinical outcome. Veterinary Pathology 2016; 53: 1117-1123. doi: $10.1177 / 0300985816638721$.

5. Scarpa F, Sabattini S and Bettini G. Cytological grading of canine cutaneous mast cell tumours. Veterinary and Comparative Oncology 2016; 14: 245-251. doi: 10.1111/vco.12090.

6. Krick EL, Billings AP, Shofer FS, Watanabe S and Sorenmo KU. Cytological lymph node evaluation in dogs with mast cell tumours: association with grade and survival. Veterinary and Comparative Oncology 2009; 7: 130-138. doi: 10.1111/j.14765829.2009.00185.x. 
7. Blackwood L, Murphy S, Buracco P, et al. European consensus document on mast cell tumours in dogs and cats. Veterinary and Comparative Oncology 2012; 10: e1-e29. doi: 10.1111/j.1476-5829.2012.00341.x.

8. Pratschke KM, Atherton MJ, Sillito JA and Lamm CG. Evaluation of a modified proportional margins approach for surgical resection of mast cell tumors in dogs: 40 cases (2008-2012). Journal of the American Veterinary Medical Association 2013; 243: 1436-1441. doi: 10.2460/javma.243.10.1436.

9. Fulcher RP, Ludwig LL, Bergman PJ, Newman SJ, Simpson AM and Patnaik AK. Evaluation of a two-centimeter lateral surgical margin for excision of grade I and grade II cutaneous mast cell tumors in dogs. Journal of the American Veterinary Medical Association 2006; 228: 210-215. doi: 10.2460/javma.228.2.210.

10. Simpson AM, Ludwig LL, Newman SJ, Bergman PJ, Hottinger HA and Patnaik AK. Evaluation of surgical margins required for complete excision of cutaneous mast cell tumors in dogs. Journal of the American Veterinary Medical Association 2004; 224: 236-240. doi: 10.2460/javma.2004.224.236.

11. Sabattini S, Scarpa F, Berlato D and Bettini G. Histologic grading of canine mast cell tumor: is 2 better than 3? Veterinary Pathology 2015; 52: 70-73. doi: $10.1177 / 0300985814521638$.

12. London CA and Seguin B. Mast cell tumors in the dog. Veterinary Clinics of North America: Small Animal Practice 2003; 33: 473-489. doi: 10.1016/S0195$5616(03) 00003-2$. 
13. Romansik EM, Reilly CM, Kass PH, Moore PF and London CA. Mitotic index is predictive for survival for canine cutaneous mast cell tumors. Veterinary Pathology 2007; 44: 335-341. doi: 10.1354/vp.44-3-335.

14. Kiupel M, Webster JD, Bailey KL, et al. Proposal of a 2-tier histologic grading system for canine cutaneous mast cell tumors to more accurately predict biological behavior. Veterinary Pathology 2011; 48: 147-155. doi: 10.1177/0300985810386469.

15. Baginski $H$, Davis $G$ and Bastian RP. The prognostic value of lymph node metastasis with grade 2 MCTs in dogs: 55 cases (2001-2010). Journal of the American Animal Hospital Association 2014; 50: 89-95. doi: 10.5326/JAAHA-MS-5997.

16. Murphy S, Sparkes AH, Blunden AS, Brearley MJ and Smith KC. Effects of stage and number of tumours on prognosis of dogs with cutaneous mast cell tumours. Veterinary Record 2006; 158: 287-291. doi: 10.1136/vr.158.9.287.

17. Kry KL and Boston SE. Additional local therapy with primary re-excision or radiation therapy improves survival and local control after incomplete or close surgical excision of mast cell tumors in dogs. Veterinary Surgery 2014; 43: 182-189. doi: 10.1111/j.1532950X.2013.12099.x.

18. Risselada M, Mathews KG and Griffith E. Surgically planned versus histologically measured lateral tumor margins for resection of cutaneous and subcutaneous mast cell tumors in dogs: 46 cases (2010-2013). Journal of the American Veterinary Medical Association 2015; 247: 184-189. doi: 10.2460/javma.247.2.184.

19. Perry JA, Culp WTN, Dailey DD, Eickhoff JC, Kamstock DA and Thamm DH. Diagnostic accuracy of pre-treatment biopsy for grading soft tissue sarcomas in dogs. 
Veterinary and Comparative Oncology 2012; 12: 106-113. doi: 10.1111/j.14765829.2012.00333.x.

20. Killick DR, Rowlands AM, Burrow RD, et al. Mast cell tumour and cutaneous histiocytoma excision wound healing in general practice. Journal of Small Animal Practice 2011; 52: 469-475. doi: 10.1111/j.1748-5827.2011.01093.x.

21. Heslin MJ, Lewis JJ, Woodruff JM and Brennan MF. Core needle biopsy for diagnosis of extremity soft tissue sarcoma. Annals of Surgical Oncology 1997; 4: 425-431. doi: 10.1007/BF02305557.

22. Stevenson M, Nunes T, Heuer C, et al. epiR: An R package for the analysis of epidemiological data 2015. R package version 0.9-69.

23. Cohen J. A coefficient of agreement for nominal scales. Educational and Psychological Measurement 1960; 20: 37-46. doi: 10.1177/001316446002000104.

24. Byrt T, Bishop J and Carlin JB. Bias, prevalence and kappa. Journal of Clinical Epidemiology 1993; 46: 423-429. doi: 10.1016/0895-4356(93)90018-V.

25. R Core Team. A language and environment for statistical computing 2016. R Foundation for Statistical Computing. http://www.R-project.org/. Accessed September 15, 2016. 


\section{Tables}

Table 1: Comparison of pre-treatment biopsy and excisional biopsy grades in 90 cases graded by both the Patnaik and the Kiupel systems.

\begin{tabular}{cccccc}
\hline & Pre-treatment biopsy & \multicolumn{3}{c}{ Excisional biopsy } \\
\hline & $\begin{array}{c}\text { Kiupel } \\
\text { low grade }\end{array}$ & $\begin{array}{c}\text { Kiupel } \\
\text { high grade }\end{array}$ & 0 & $\begin{array}{c}\text { Kiupel } \\
\text { low grade }\end{array}$ & $\begin{array}{c}\text { Kiupel } \\
\text { high grade }\end{array}$ \\
\hline $\begin{array}{c}\text { Patnaik } \\
\text { grade I }\end{array}$ & 5 & 9 & $\begin{array}{c}\text { Patnaik } \\
\text { grade I }\end{array}$ & 1 & 0 \\
\hline $\begin{array}{c}\text { Patnaik } \\
\text { grade II }\end{array}$ & 73 & 3 & $\begin{array}{c}\text { Patnaik } \\
\text { grade II }\end{array}$ & 70 & 16 \\
\hline $\begin{array}{c}\text { Patnaik } \\
\text { grade III }\end{array}$ & 0 & $\begin{array}{c}\text { Patnaik } \\
\text { grade III }\end{array}$ & 0 & 3 \\
\hline
\end{tabular}

Table 2: Multivariable logistic regression model outputs of the association between concordance of mast cell tumor grades on pre-treatment biopsy and excisional biopsy, by grading system.

\begin{tabular}{llllll}
\hline Grading scheme & Variable $^{*}$ & OR & SE & (95\% CI) & P-value \\
\hline \multirow{2}{*}{ Patnaik } & age & 0.61 & 0.16 & $(0.37,1.01)$ & 0.056 \\
& $\log _{2}$ (tumor size) & 6.99 & 7.21 & $(0.93,52.7)$ & 0.059 \\
& & & & & \\
Kiupel & age & 0.79 & 0.13 & $(0.38,1.08)$ & 0.14 \\
& $\log _{2}$ (tumor size) & 1.16 & 0.53 & $(0.47,2.84)$ & 0.75 \\
& & & & & \\
(Units: age (years); tumor size (mm). OR = odds ratio; SE = standard error; CI = confidence \\
interval)
\end{tabular}

This article is protected by copyright. All rights reserved. 


\section{University Library}

\section{- M M N E R VA A gateway to Melbourne's research publications}

Minerva Access is the Institutional Repository of The University of Melbourne

Author/s:

Shaw, T;Kudnig, ST;Firestone, SM

Title:

Diagnostic accuracy of pre-treatment biopsy for grading cutaneous mast cell tumours in dogs

Date:

2018-06-01

Citation:

Shaw, T., Kudnig, S. T. \& Firestone, S. M. (2018). Diagnostic accuracy of pre-treatment biopsy for grading cutaneous mast cell tumours in dogs. VETERINARY AND COMPARATIVE ONCOLOGY, 16 (2), pp.214-219. https://doi.org/10.1111/vco.12346.

Persistent Link:

http://hdl.handle.net/11343/293386 